\title{
Participación Infantil en la Transformación de sus Espacios de Aprendizaje: Democratizando la Creación mediante un Proyecto de Fabricación Digital en un
} Fablab

\author{
Child Participation in the Transformation of their Learning \\ Spaces: Democratizing Creation through a Digital Fabrication \\ Project in a Fablab
}

\section{Participação Infantil na Transformação de Espacios de Aprendizagem: Democratizando a Criação através de um Projeto de Fabricação Digital em um Fablab} Moisés Esteban-Guitart ${ }^{2}$ Sara San Gregorio ${ }^{3}$

${ }^{1}$ Universidad Autónoma de Madrid. ${ }^{2}$ Universitat de Girona. ${ }^{3}$ Medialab-Prado Madrid

\begin{abstract}
Se presenta un proyecto participativo incluido en la programación de aula de dos grupos de educación primaria de un centro escolar situado en Madrid. El objetivo es situar y señalar ciertas particularidades metodológicas de este estudio de caso, un proyecto de investigación acción de orientación transdisciplinar que ha implicado a la comunidad educativa del colegio, a investigadores y a profesionales de una institución cultural de reconocido prestigio internacional, con la pretensión de promover el interés y la relevancia de una investigación educativa basada en las prácticas que puede ayudar a intervenir e innovar en procesos de cambio educativo, al mismo tiempo que genera conocimiento científico, dando importancia al contexto local y a las diferencias de cada comunidad.
\end{abstract}

Descriptores: Investigación participativa, Aprendizaje activo, Fabricación asistida por ordenador, Innovación educacional, Instalación educativa

\begin{abstract}
This paper introduces a participatory project included in the classroom programming of two groups of primary education in a school situated in Madrid. The main interest is to situate and mark certain methodological particularities of this case study, a transdisciplinary action research project that has involved the educational community of the school, researchers and professionals of a cultural institution with international prestige, with the aim of promoting the interest and relevance of practice-based educational research that can help to intervene and innovate in educational change processes, while generating scientific knowledge, giving importance to the local context and the differences of each community.
\end{abstract}

Keywords: Participatory research, Experiential learning, Computer aided manufacturing, Educational innovations, Educational facilities

*Contacto: javier.gonzalezpatino@uam.es

ISSN: 2254-3139

www.rinace.net/riejs/

revistas.uam.es/riejs
Recibido: $\quad 15$ de diciembre 2016

$1^{\text {a }}$ Evaluación: 13 de enero 2017

$2^{\text {a }}$ Evaluación: 23 de febrero 2017

Aceptado: $\quad 4$ de marzo 2017 
Este artigo apresenta um projecto participativo incluso na programação das aulas de dois grupos de alunos de educação primária de um centro escolar em Madrid. O objectivo é assinalar e indicar algumas particularidades metodológicas deste estudo, um projeto de investigação e de orientação interdisciplinar que tem envolvido a comunidade educativa do colégio, além de pesquisadores e profissionais de uma instituição cultural de reconhecido prestígio internacional. O objectivo é o de promover o interesse na pertinência de uma pesquisa educativa baseada nas práticas que podem ajudar a intervir e inovar os processos educativos ao mesmo tempo que geram conhecimento científico, dando importância ao contexto local e as diferenças de cada comunidade.

Palavras-chave: Pesquisa participativa, Aprendizagem activa, Fabricação assistida por computador, Inovações educacionais, Instalações educacionais.

\section{Introducción}

La investigación sobre el impacto evolutivo y social de los desarrollos tecnológicos, especialmente el referido a los efectos que podrían ejercer los cambios culturales sobre la infancia, tiende a producir de un tiempo a esta parte un discurso científico que destaca el potencial creativo, de manera más o menos implícita, de la tecnología (móviles, videojuegos o internet) argumentando que puede contribuir decisivamente al desarrollo social e intelectual suponiendo una ayuda para estudiar y preparar a los niños para su futuro profesional (Marsh y Millard, 2006; Plowman, McPake y Stephen, 2010). Este análisis se ve matizado por la falta de apoyo que provocan ciertas medidas gubernamentales a la hora de plantear la educación de los niños en la economía del conocimiento y las preocupaciones a largo plazo por la brecha digital. Los medios de comunicación también tienden a producir un discurso que resalta los riesgos de la tecnología frente a las evidencias a favor (Boyd, 2014; Jenkins, Itó y Boyd, 2015). Sin embargo, la revolución tecnológica que estamos viviendo lo hace mediando en una gran revolución social, sobre todo en lo que se refiere a los medios de comunicación y a las posibilidades que nos aporta una tecnología que ha provocado una transformación tan profunda en la manera en la que gestionamos la información y producimos conocimiento, adentrándose tanto en el hogar como en el trabajo o en la escuela (Castells, 2009).

Resulta especialmente relevante para contextualizar el proyecto presentado en este artículo, llamar la atención sobre la relación que se establece entre educación y tecnología, y de forma más precisa entre procesos de fabricación y la institución educativa, pues es justamente durante la anterior gran revolución tecnológica de la historia de la humanidad, la industrial, que coloca en el centro de la economía a las fábricas, cuando surge la escuela moderna tal como la conocemos, como un sistema de escolarización universal. En función de los avances que realizamos, cada cierto tiempo nuevas habilidades y actividades intelectuales se vuelven esenciales para el trabajo y la convivencia. Esa correspondencia o carrera entre medios de producción y enseñanza, y la necesidad de ajuste social para alcanzar una fase de prosperidad en la que lo educativo no se vea superado por las posibilidades de los logros tecnológicos (Goldin y Katz, 2010), está en el trasfondo del interés de la comunidad educativa que participa en este estudio: un grupo de niños, sus profesores, familias y los mediadores de una acreditada institución dedicada a la cultura digital de la ciudad de Madrid, que se propusieron descubrir y aplicar procesos de diseño y fabricación digital con la intención de mejorar su centro escolar. La cultura maker y la fabricación digital habilitan una ocasión para aportar ideas, alfabetización y herramientas creativas a la infancia (democratizando 
saberes, tareas y competencias, previamente reservados para los expertos), que a través del trabajo por proyectos, el construccionismo o la agencia del aprendiz, nos permite articular las necesidades de innovación educativa aplicando las propuestas teóricas de autores como John Dewey, Lev Vygotski, Paulo Freire, Urie Bronfenbrenner, David Ausubel o Seymour Papert (Blikstein, 2013).

El interés investigador de este trabajo es entender las lógicas de lo digital como una forma de estar y dar sentido al mundo en el que vivimos (Horst y Miller, 2012), de manera que los aportes teóricos y metodológicos ayuden a los participantes y a los agentes de socialización (familias, profesores, comunidad, medios de comunicación, etc.) a analizar, interpretar y mejorar su realidad social, y que compartir esta experiencia pueda servir como inspiración a otros dando importancia tanto a sus particularidades como al contexto local. Sería difícil imaginar mejor momento histórico para aspirar al cambio educativo para la justicia social que el actual, de convergencia mediática, protagonizado por una sociedad hiperconectada (Van Dijck, 2013) que está transformando los usos de los medios de comunicación mediante el auge de la cultura participativa y creando nuevos estilos de democratización de la experiencia y la creación (Jenkins, 2008; Jenkins, Ford y Green, 2013). La investigación acción aparece por tanto como un enfoque relevante para una investigación como ésta basada en las prácticas y con una visión de la educación a largo plazo que, alejándose del modelo dominante de búsqueda y propuesta de las "mejores prácticas" se acerque a otro más respetuoso de "prácticas bastante buenas" (Erickson, 2014) que persiga la incorporación de la agencia y autonomía de la comunidad educativa expandida por este empoderamiento digital. Dado el enfoque y objetivos de este estudio se ha concedido bastante importancia a la innovación que parte de las prácticas sociales y culturales de la comunidad constituida con el ánimo de explorar e investigar, a su vez, el impacto de dichas prácticas. Por otro lado, y aunque el ámbito educativo del que parte es formal, una aproximación de tipo aplicado que además conecta con contextos no formales e informales (Cobo y Moravec, 2011) es compatible y resulta más propicia para la creación y funcionamiento de una comunidad de práctica (Wenger, 2005; Wenger, McDermott y Snyder, 2002) que permita analizar posteriormente las dinámicas y complejidad de una situación basada en una actividad de aprendizaje eminentemente situada (Lave y Wenger, 1991).

\section{Perspectivas actuales de la investigación acción}

La investigación acción participante o participativa (IAP) combina inseparablemente la creación de conocimiento y la intervención social (Regina Frizzo, 2008). Es una forma de concebir la investigación en la que la participación en el proceso de los sujetos observados, se considera una oportunidad para promover la comprensión que estos tienen de su realidad social con la intención de que la transformen colectivamente.

En los años 40 del siglo Xx, Kurt Lewin rompe con la tradición positivista de separar el papel de la ciencia del orden práctico del mundo, promoviendo con sus trabajos un buen número de estudios en la década de los 60 tanto en Latinoamérica como en Europa y Estados Unidos. Una década después, y principalmente en Latinoamérica donde varios países sufrían regímenes dictatoriales, la IAP se identifica con un activismo militante, tomando una posición ideológica y política a favor de grupos oprimidos que participan, independientemente de su grado de educación y estatus, contribuyendo de forma activa al proceso de investigación (Freire, 1970). La formulación actual de la IAP llegó con la creación del centro de investigación y acción social de Fals Borda y colaboradores 
(1972). Basado en la inserción del investigador en la comunidad, diseñaron un método, la investigación militante, que analizaba condiciones históricas y estructura social, el desarrollo del nivel de conciencia de sus miembros y de organizaciones políticas y grupos de acción, y se caracterizaba por su énfasis en la solución de problemas y el compromiso con la comunidad.

A partir de la década de los 80 a las aportaciones de la psicología social y comunitaria se unirán las de otros investigadores vinculados a trabajos con intereses diferentes a los de las clases sociales menos favorecidas, lo que no hará sino fortalecer aún más esta corriente (Balcazar, 2003; Regina Frizzo, 2008). Es precisamente dentro de este encuadre de la IAP en el que se situaría este trabajo, y los aspectos conceptuales centrales a la hora de plantearlo serían:

- Legitimar las prácticas sociales y culturales como un elemento para generar conocimiento científico, tanto para identificar problemas o preguntas de investigación como para plantear soluciones.

- Eliminar la separación convencional entre investigador y participante, pues únicamente mediante la interacción entre ambos es posible el proceso de investigación e intervención.

- Determinación de producir cambios tanto en la situación (práctica educativa) como en los participantes e investigadores (comunidad de práctica, conocimiento producido y relaciones establecidas), fruto de un proceso continuo de acción-reflexión.

\section{Método}

La experiencia de aprendizaje presentada en esta publicación se creó con la intención de que unos alumnos de educación primaria conocieran los procesos de diseño y fabricación digital. Formó parte de un programa más amplio, el proyecto Arcángel :D, promovido en su centro escolar por Mediática, accesible en http://mediatica.co (Mediática, 2016a), colectivo fundado por el primer autor de este artículo y que realiza labores de acompañamiento y experimentación con comunidades educativas que desean producir cambios en sus usos y prácticas digitales, cuyo enfoque metodológico es la investigación acción y el modo de implicación el de la consultoría participativa (Wardrop y Withers, 2014).

La propuesta incluyó el diseño del plan de actuación para la creación de una comunidad educativa expandida (González-Patiño, 2015) sobre la base de una comunidad de aprendizaje, en colaboración con la Fundación Maestro y el claustro de profesores del colegio concertado Arcángel de Madrid, la Asociación de Madres y Padres de Alumnos (AMPA) y Escala Digital, proyecto de investigación de Sara San Gregorio en MedialabPrado, institución dedicada a la cultura digital perteneciente al Área de Gobierno de Cultura y Deportes del Ayuntamiento de Madrid. La figura 1 sintetiza la complejidad de contextos, roles de los participantes e instituciones implicadas, incorporando incluso actividades de transferencia de conocimiento como la realización por parte de los niños de un taller dirigido a adultos (Flipped workshop) que se celebró dentro de un congreso internacional dedicado al juego de construcción. Como en otras colaboraciones de Mediática con comunidades o colectivos (centros educativos, grupos de investigación, 
empresas, instituciones, etc.), se trataba de proporcionar inspiración para innovar en las prácticas de aprendizaje o creación de conocimiento, explorando y aprovechando las trazas o características de la cultura de nuestra sociedad conectada, y abarcando, con una orientación transdisciplinar y sistémica, las vertientes aplicadas y de investigación de la disciplina temática que de un tiempo a esta parte se viene identificando como medios digitales y educación (digital media and learning), vinculada a dinámicas de aprendizaje y socialización con mediación digital (Gee, 2013).

El resultado que se persigue es establecer las bases para que la comunidad de aprendizaje produzca sus propios progresos con la mayor autonomía posible. El modelo metodológico tiende a la organización del trabajo por proyectos y a realizar actividades con carácter colaborativo y abierto mediadas por aplicaciones de la web social que permitan el intercambio, la difusión, el debate y la recuperación posterior del conocimiento generado por los participantes (González-Patiño y Esteban-Guitart, 2014).

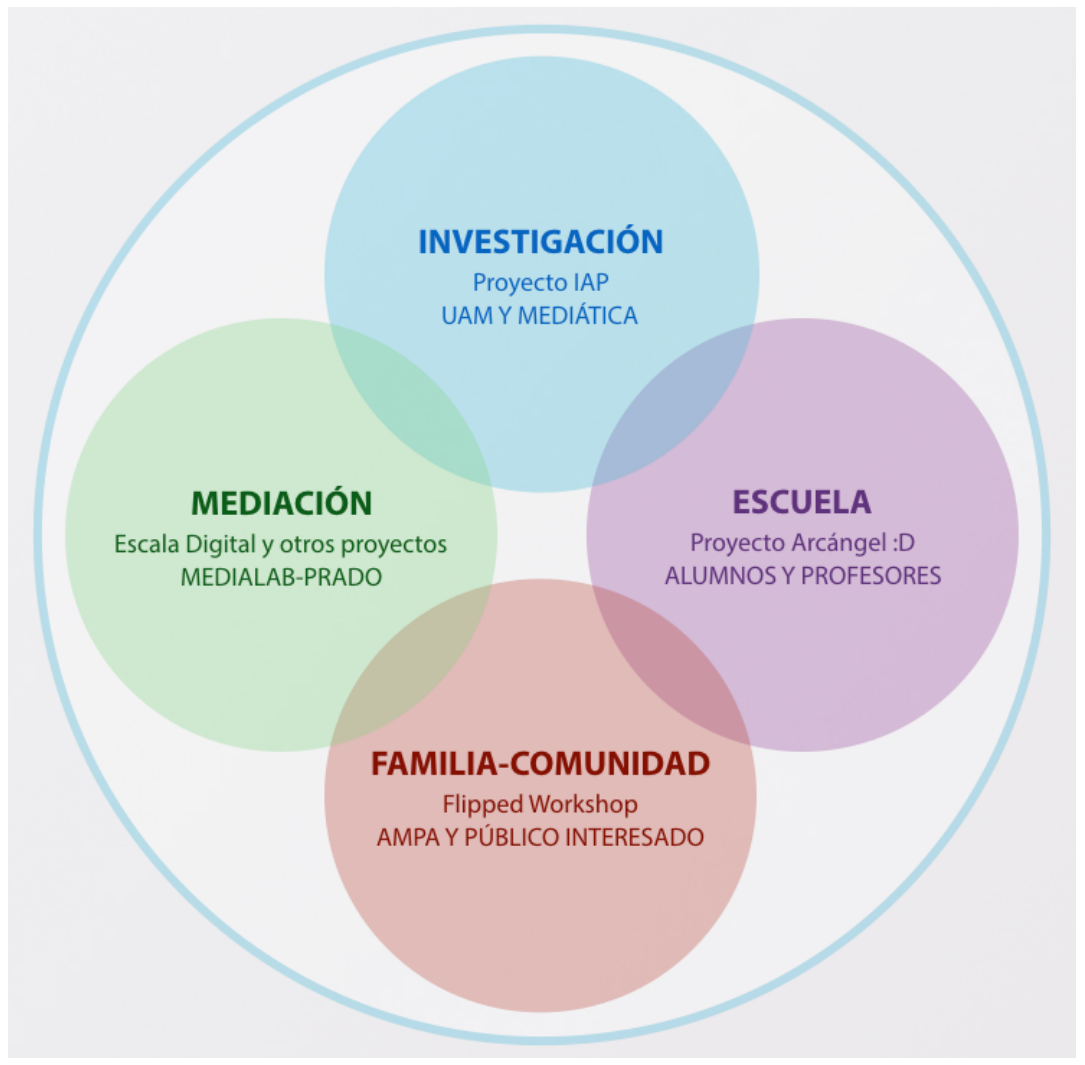

Figura 1. Estructura de participación del proyecto Arcángel :D

Fuente: Elaboración propia.

La producción de conocimiento de esta IAP partió del interés del primer autor en promover cambios y mejoras en la comunidad educativa a la que pertenece como padre de una alumna. Según las prácticas propias de la IAP, los objetivos y las preguntas de investigación se negocian, se replantean y en ocasiones surgen, como consecuencia del propio proceso de intervención y la interacción de los actores participantes. De este modo, no se hizo ninguna propuesta ni intervención hasta que se llevó a cabo un análisis de necesidades conjunto con los profesores, familias y los otros investigadores externos (entre otros Cronicalab, un grupo de trabajo de Medialab-Prado dedicado a documentar 
audiovisualmente las iniciativas en las que colabora la institución), con el fin de evitar posteriores imposiciones, así como, caer en procesos circulares que no aclaran nunca las metas (Castellá Sarriera, 2008).

\subsection{Contexto de la investigación}

La mayor parte del trabajo de campo ha tenido lugar durante el primer semestre del año 2016, realizado con dos grupos de educación primaria, de tercer y cuarto curso, de un colegio concertado laico del distrito de Retiro de la ciudad de Madrid. El centro goza de cierto reconocimiento por su sensibilidad hacia las prácticas docentes que favorecen el protagonismo, la autonomía y la implicación del alumno, y es conocida asimismo la elevada implicación de las familias que forman parte de su comunidad educativa. A pesar de no estar inscrito como centro bilingüe de la red de la Comunidad Autónoma de Madrid, desarrolla un proyecto propio de aprendizaje de la lengua inglesa con dedicación horaria y recursos equivalentes a los del programa oficial. La población que atiende resulta bastante homogénea en términos socioeconómicos, étnicos y culturales, y por sus características arquitectónicas de accesibilidad reducida, se encuentra en una colonia de viviendas individuales ocupando dos edificaciones con varias plantas adaptadas para acoger una institución educativa, y su tamaño, de línea uno tanto en la etapa de infantil como de primaria, tampoco cuenta con medidas de integración ni capacidad para atender a niños con necesidades educativas especiales. Los alumnos en su mayoría son de nacionalidad española aunque también acoge algunos niños de otras procedencias; un porcentaje elevado son hijos de titulados superiores aunque no pertenecientes a élites económicas como ocurre en otros centros de la zona, donde además predominan los colegios religiosos.

En cuanto a equipamiento tecnológico se refiere, el centro dispone de una sala compartida de informática con ordenadores de sobremesa conectados a internet y red wifi, aunque la capacidad de la conexión es limitada. Todos los profesores tienen ordenador en su aula y pizarra digital interactiva. No utilizan libros de texto salvo para contenidos o actividades concretas (como para la enseñanza de la lengua inglesa), y recurren de manera puntual y suplementaria a materiales didácticos en formato digital y accesibles a través de internet (comercializados por las editoriales generalmente bajo la denominación de aula virtual o similar), ya que parte del claustro y de las familias no considera que el uso de herramientas digitales sea coherente con la ideología educativa del colegio, asociándolas con posibles efectos negativos (como el riesgo de favorecer el acoso o perjudicar la concentración). Llama por tanto la atención, esta aparente contradicción entre el compromiso con una metodología innovadora en lo docente y el escaso o poco definido papel que juegan las tecnologías de la información y la comunicación a pesar de que España presenta el mayor grado de adopción en dispositivos como el smartphone con el $82,8 \%$, la tablet con el $58,9 \%$, o el e-reader con el 37,4\% (Fundación Telefónica, 2016).

\subsection{Procedimiento}

La complejidad estructural de la participación en este estudio, así como la confluencia de intereses investigadores, permitió conseguir un amplio y modalmente diversificado corpus de datos que provee un buen número de abordajes analíticos posibles. Este artículo, al tratarse de la primera publicación sobre el mismo, pretende centrarse en situar la investigación y presentar ciertas particularidades sobre diseño y metodología. De acuerdo con los objetivos descritos y las posibilidades que surgieron, la obtención de 
datos implicó una selección de técnicas en la que predomina una metodología etnográfica para la recogida (Lancy, Bock y Gaskins, 2010), investigación y análisis visual (González-Patiño, 2011; González-Patiño y Esteban-Guitart, 2015; Mitchell, 2011; Pink, 2013; Thomson, 2010), etnografía del espacio virtual, observación participante en las sesiones de trabajo y, entrevistas semiestructuradas y conversaciones informales con los niños, profesores, talleristas y familias. La comunidad educativa expandida creada, proyecto Arcángel :D, implementó un nodo central o hub, un sitio web que integra la participación y comunicación, accesible en http://arcangel.mediatica.co/ (Mediática, 2016b) y que junto a los mensajes de la lista de correo del grupo, constituyeron una importante fuente de conversaciones mediadas digitalmente. Asimismo, se recogieron datos de diversas fuentes complementarias (observaciones informales, documentación y mantenimiento del $h u b$, participación en eventos, etc.).

Durante el proceso de análisis de necesidades se planteó la idoneidad de trabajar en colaboración con una institución pública con experiencia en el prototipado de proyectos de cultura digital como Medialab-Prado Madrid. A pesar de que al menos dos grupos de investigación de la misma compartían intereses con el proyecto iniciado por Mediática en el colegio Arcángel, un aspecto conceptual básico es el que se refiere a la definición de necesidad. La actividad de análisis para especificar los problemas, expectativas, demandas o preocupaciones de la comunidad es continua a lo largo de la IAP, pues se trata de transformar las necesidades percibidas (cognición) en necesidades sentidas (añadiendo afecto o concienciación). Hay que tener en cuenta que en ocasiones la comunidad percibe una necesidad pero no la siente, bien porque está insensibilizada (por las ideologías educativas de ciertos miembros influyentes o por los medios de comunicación) o bien porque ha naturalizado las preocupaciones del investigador principal (que en este caso aúna varios roles que pueden entrar en conflicto, como el de padre, asesor y científico). Además de la distinción entre necesidades sentidas y percibidas, se podría matizar entre percibidas (subjetivas) e inferidas (más objetivas y referidas a cuestiones normativas) que provienen de criterios preestablecidos o de profesionales e instituciones vinculadas a la comunidad (como la inspección de la Consejería de Educación autonómica). Incluso podríamos distinguir entre necesidades (como las carencias tecnológicas) y problemas (conflicto sobre la idoneidad de la adquisición de ciertas competencias digitales). En cualquier caso, fueron los investigadores los que determinaron lo que se entendía por necesidad, estando atentos a las atribuciones que la comunidad hizo de su significado (Castellá Sarriera, 2008). En conclusión, los aspectos considerados en el análisis de necesidades fueron:

- Las necesidades sentidas, percibidas e inferidas por los actores integrantes de la comunidad: desarrollar una experiencia de aprendizaje situado aprovechando los recursos de diseño participativo y fabricación digital del fablab de Medialab-Prado, para producir cooperativamente una transformación en la forma de trabajar y aprender en dos grupos de primaria del colegio Arcángel.

- Los problemas y las formas de resolverlos que proponía la comunidad: limitaciones materiales y de tiempo de los alumnos, profesores, investigadores y equipo de mediación.

- Y los recursos que poseía o a los que podía acceder: accesibilidad al fablab e internet, conocimientos tecnológicos y apoyo para dinamizar las diferentes sesiones. 


\section{Resultados}

Una vez completado el análisis se diseñó el plan de acción, la intervención, que consistió en un proyecto que se extendería a lo largo de la segunda mitad del curso y en el que estarían implicados dos grupos de primaria para descubrir y aplicar procesos de diseño participativo y fabricación digital, con la intención de producir un objeto con capacidad mediadora en alguna mejora educativa para su centro escolar. A continuación se presentan las fases que se ejecutaron y sobre las que se realizó la recogida de datos, aclarando previamente que algunas implicaron varias sesiones de trabajo. Otra de las características fundamentales de la IAP, que está presente en todas las dinámicas que se llevaron a cabo, es la relativa a las cuestiones de agencia de los participantes, especialmente de los niños, planteando como prioridad el empoderamiento de los actores implicados para crear conocimiento mediante la comprensión de su realidad social e intentar transformarla de forma colectiva.

\subsection{Descubriendo la tecnología para la fabricación digital}

La primera sesión consistió en visitar el fablab de Medialab-Prado Madrid. Además de presentar el laboratorio de fabricación y su aparataje, y de reflexionar sobre el posible impacto de lo digital en los modelos de producción y consumo conocidos hasta ahora, se explicaron las cuestiones generales del proyecto y sus objetivos. Junto a los alumnos y profesores, asistieron también algunas familias. Para promover la búsqueda de sentido de la experiencia (aprendizaje significativo) se hizo coincidir con la exposición "Objetos comunes" que recogía una selección de objetos construidos por distintas comunidades en Medialab-Prado en base a la fabricación distribuida y personalizada característica del conocido como movimiento maker, responsable del auge de los fablabs (Blikstein, 2013).

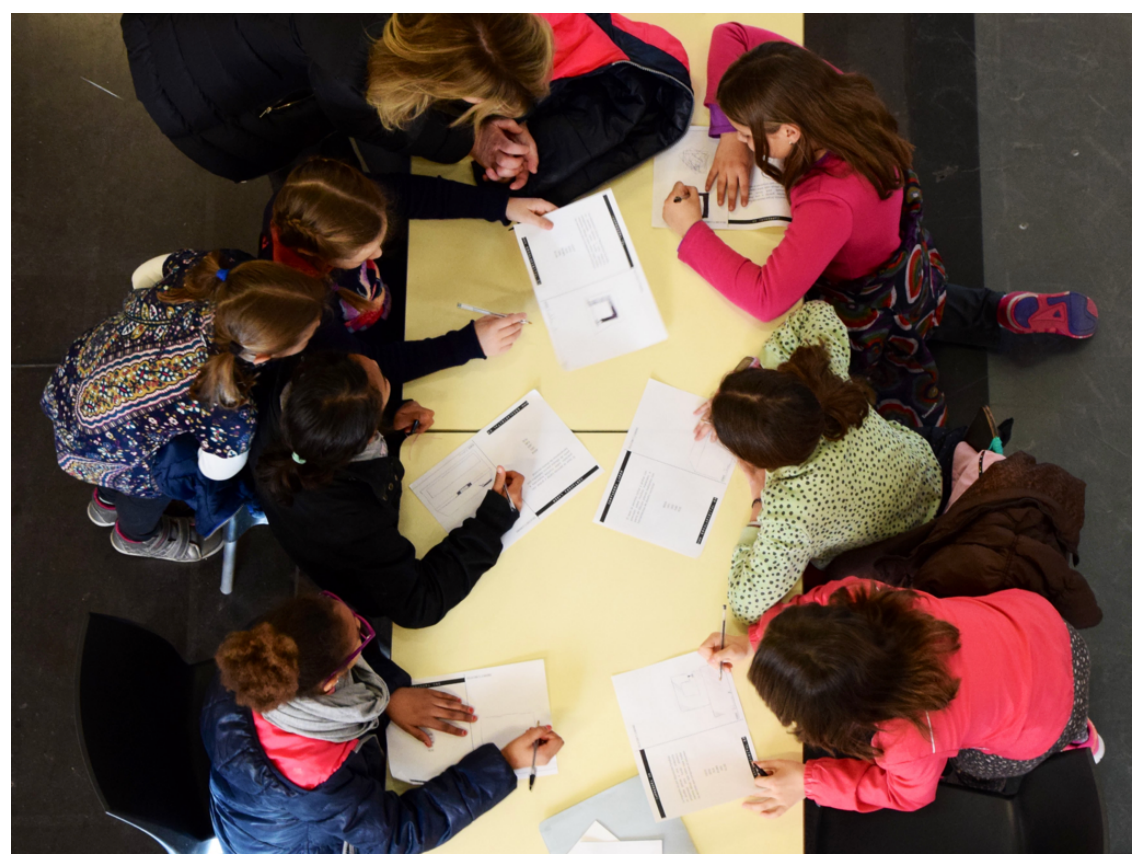

Figura 2. Descubriendo y dibujando las máquinas del fablab Fuente: Elaboración propia.

La visita se desarrolló distribuyendo a los alumnos según tres condiciones, un grupo que dibujó las máquinas después de verlas y se las explicó a otro que las tuvo que dibujar 
antes de que se las enseñaran, y un tercer grupo que dibujó las máquinas sin verlas previamente ni haber recibido explicación alguna. La figura 2 ilustra el funcionamiento de esta actividad. El análisis de los dibujos permitió evaluar los conocimientos previos. Posteriormente todos los grupos identificaron con qué máquinas de fabricación digital habían sido producidos los objetos de la exposición.

\subsection{Empoderamiento investigador}

El primer paso de un proyecto con el que se pretende diseñar y fabricar un objeto es determinar cual. Teniendo presente que el objetivo era lograr una mejora en la forma de trabajar y aprender en el colegio, y la importancia dada a la autonomía y la participación, la siguiente fase consistió en ayudar a los niños a realizar el análisis de sus propias necesidades y deseos, proponer soluciones y elegir después una por consenso porque los recursos disponibles solo permitían producir el prototipo de un objeto. Elaboraron individualmente un registro mediante la primera herramienta del proceso de diseño participativo, un cuadernillo que denominamos el "ideatorio", que recogía las consignas para observar, proponer y proyectar ideas. Durante cuatro días anotaron aquello que habían identificado como mejorable en el colegio y una propuesta de cambio. El quinto día eligieron una de las propuestas haciendo un boceto dibujado del objeto que querían fabricar.

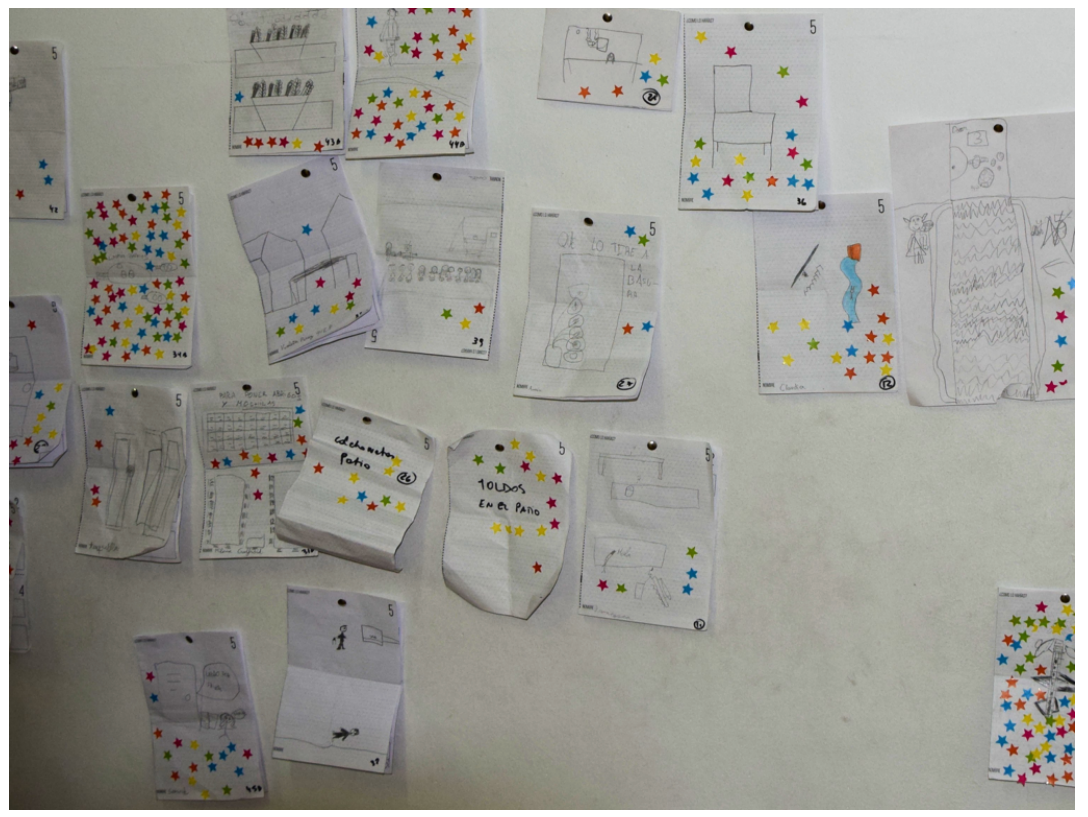

Figura 3. Votación de propuestas para consensuar un proyecto común Fuente: Elaboración propia.

En una sesión posterior celebrada en el centro escolar, se presentaron y votaron todas las propuestas para seleccionar el proyecto. Se entregaron 15 adhesivos de color a cada participante, tres pegatinas de entre cinco colores, para valorar los bocetos que se colocaron en un mural (figura 3). Se asignaron los colores a las siguientes categorías: verde para la idea que más problemas resuelve, naranja para la más bonita, amarilla para la más divertida, azul para la más viable y rosa para la más fácil de replicar en un fablab. Como resultado del proceso acordaron diseñar y crear los muebles que más tiempo utilizan cuando están en el colegio, las mesas y sillas de clase, identificando entre sus necesidades la de incorporar nuevas funcionalidades que incluyan soluciones y prácticas 
complementarias (adaptables, que les ayuden a mejorar cuestiones de almacenamiento, posibilidad de efectuar cambios de distribución en el aula en función de la actividad que estén desarrollando, y un largo etcétera).

\subsection{Proceso de diseño participativo}

Una vez definido el reto, la creación y coordinación del plan de diseño y fabricación digital fue una de las aportaciones clave de la investigadora de Medialab-Prado y arquitecta, Sara San Gregorio. En los últimos años son cada vez más los ámbitos profesionales (ingeniería, arquitectura y diseño entre otros) que valoran y utilizan procedimientos de pensamiento de diseño o design thinking por su eficacia para crear conocimiento situado o encontrar soluciones plausibles, explorando y replanteando problemas poco o incluso mal estructurados. Sara expuso a la comunidad el interés por aplicarlo en una dinámica de enseñanza-aprendizaje y tanto los profesores como el resto de investigadores apoyaron la idea de construir un estudio de caso que busque evidencias sobre la contribución del design thinking al desarrollo de las capacidades creativas y adaptativas de los estudiantes, permitiéndoles adquirir conocimientos y habilidades, y desarrollar competencias necesarias para la resolución participativa de problemas complejos (Koh y colaboradores, 2015; Scheer, Noweski y Meinel, 2012).

Los niños trabajaron colaborativamente para hacer una síntesis de sus ideas y reconceptualizar el mobiliario personal de clase (mesa, silla y espacio de almacenaje). Tras la selección del proyecto y antes de pedirles que elaboraran las maquetas de sus diseños, en una sesión de discusión de ideas se detectó la necesidad de ayudarles en el proceso de investigación y búsqueda de referencias, puesto que las mejoras que propusieron basadas únicamente en su conocimiento previo resultaron bastante canónicas o convencionales. Al presentar el contexto de la investigación se señalaba que uno de los objetivos fundamentales del proyecto Arcángel :D, dentro del cual se llevó a cabo esta actividad de design thinking, era el de inspirar nuevas prácticas de aprendizaje que permitieran a profesores y alumnos desarrollar sus competencias digitales, por lo que se decidió utilizar una plataforma de la web social con este propósito. En concreto, los profesores, alumnos, familias, investigadores y talleristas (colaboradores provenientes de instituciones culturales vinculadas principalmente con el arte y la arquitectura que se incorporaron en esta fase) compartieron referencias de diseño creando un tablero en la red social Pinterest (figura 4) que se presenta como un catálogo de ideas global y que permite además de organizar material visual colaborando online, recibir recomendaciones basadas en las búsquedas y clasificación de todos sus usuarios, facilitando el acceso a una gran cantidad de información relevante. La selección de referencias fue utilizada para crear un andamiaje que facilitara decisiones y actividades de las siguientes etapas del proceso de diseño y fabricación, como por ejemplo, incluir ideas de muebles de autoconstrucción por piezas que por un lado fueran reproducibles con facilidad con la maquinaria de un fablab y por otro permitieran distintas alternativas de montaje para explorar una gran variedad de usos y aplicaciones. 


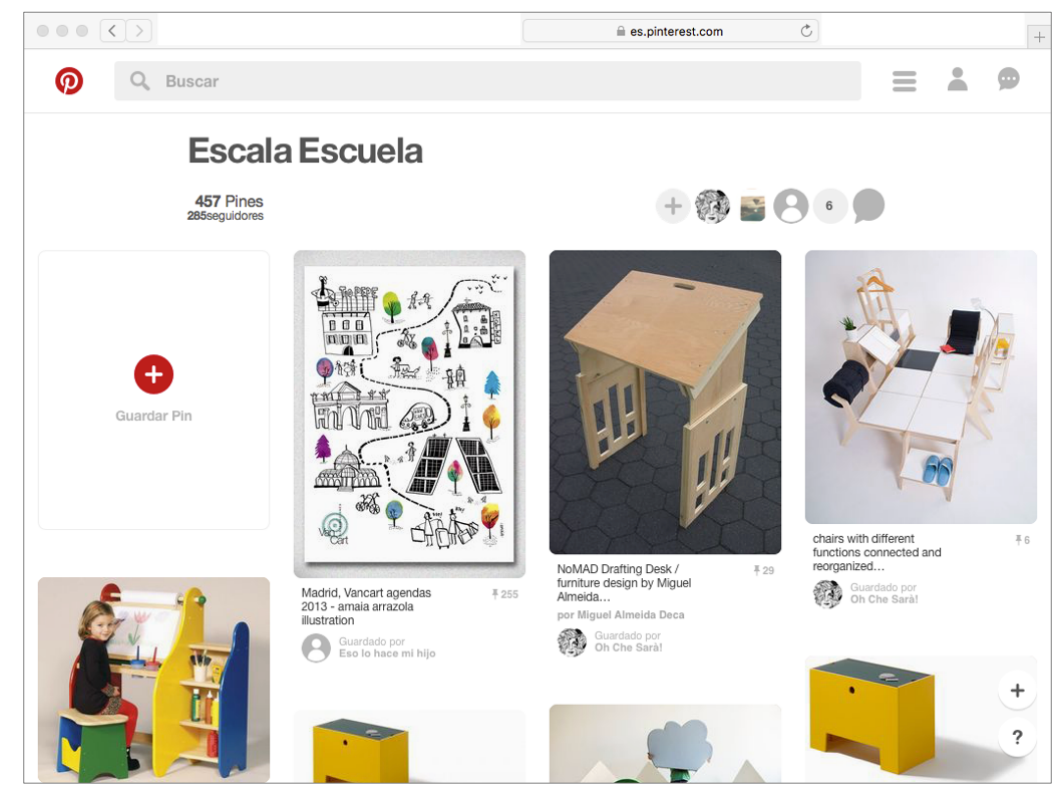

Figura 4. Tablón de la red social Pinterest para buscar y compartir referencias Fuente: Elaboración propia.

El siguiente paso consistió en producir con la cortadora láser una maqueta de cartón a escala de una serie de piezas de mobiliario que combinaban geométricamente con las mesas del aula y que ayudaban a solucionar las necesidades identificadas (como la adaptabilidad o el almacenamiento de objetos de uso cotidiano), pero sobre todo, provocaban la exploración de nuevas distribuciones de espacio relacionadas con distintas situaciones lectivas (clase magistral, trabajo en grupo, etc.). A lo largo del proceso se produjeron aprendizajes de muy diverso tipo (curriculares, procedimentales, valores, etc.), encontrando los profesores numerosas oportunidades para dotar de significado y situar su actividad docente:

\begin{abstract}
Ayer estuvimos trabajando en clase cómo representar un objeto a escala, mantener las proporciones. Después de un rato con dibujos de polígonos, me di cuenta que algunos no se enteraban... entonces recordé que teníamos pendiente lo de medir la clase y dibujar el plano para colocar las mesas y hacer pruebas, asi que nos pusimos a hacerlo y no necesité explicarles nada más. (Extracto 1. Conversación con Javier, tutor del grupo de cuarto de primaria)
\end{abstract}

\title{
3.4. Exploración de usos: talleres de autoconstrucción
}

La colección de piezas a escala funcionaba a modo de juego para que los niños buscaran alternativas de transformación de uno de los espacios que habitan, el aula, pero como resultado de un procedimiento de diseño participativo también se convirtió en la ocasión para aparecer ante los profesores, familias y otros adultos como agentes de cambio (figura 5). Aprovechando la celebración de un encuentro de debate y trabajo sobre el juguete de construcción que se celebró en Medialab-Prado Madrid en mayo de 2016, "Construcciones en juego", los alumnos del colegio Arcángel intervinieron como talleristas dinamizando una sesión de juego en la que fueron los adultos los que siguieron sus consignas y realizando un ejercicio de transferencia de resultados de su trabajo de investigación. 


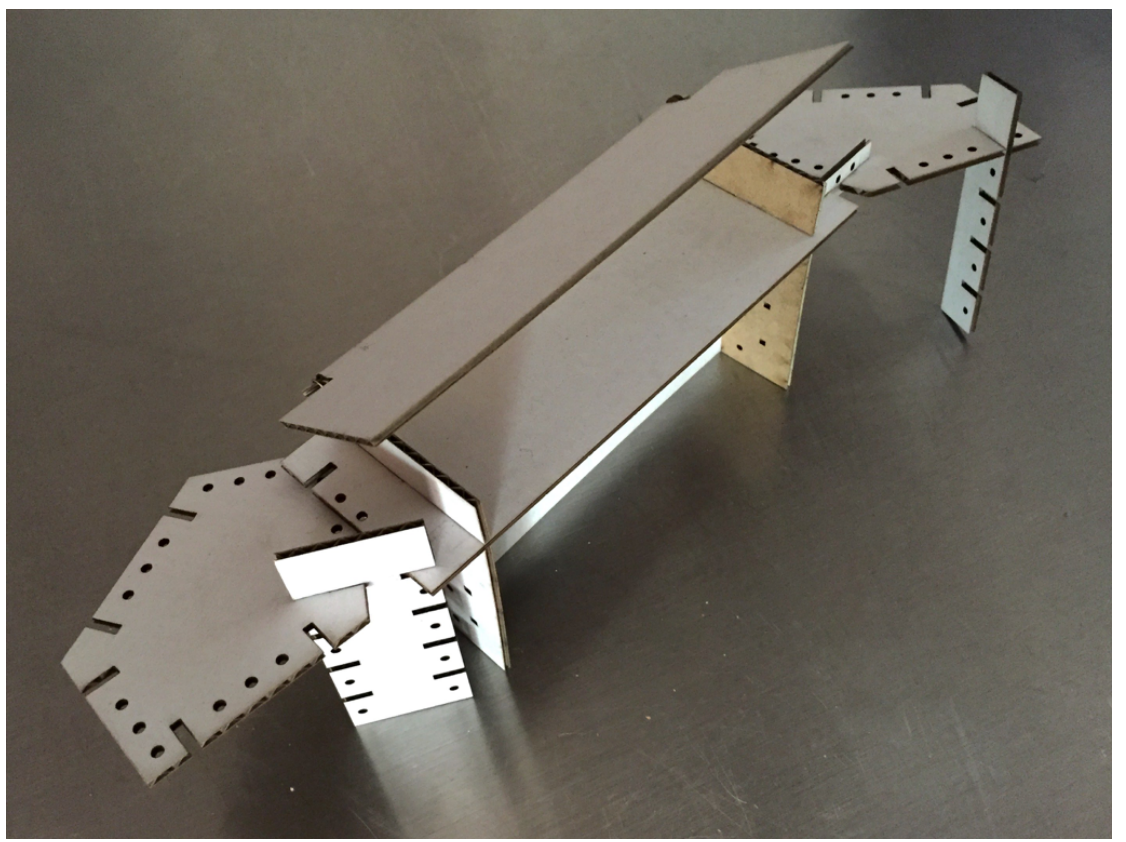

Figura 5. Maqueta a escala, con piezas de cartón, para construir el mobiliario de aula Fuente: Elaboración propia.

\subsection{Fabricación del prototipo final y valoración}

En la última sesión de trabajo en el fablab, y una vez incorporados al diseño asistido por ordenador ciertos ajustes y modificaciones de la maqueta a escala, se fabricó el prototipo final con la fresadora utilizando tableros de madera de densidad media (DM). A continuación, se realizó una actividad de juego con las piezas a tamaño real que sirvió para confrontar el trabajo de ideación y diseño con las posibilidades reales de uso, intentando equilibrar el balance entre instrucción y construcción para que los alumnos experimentasen sus aprendizajes de manera situada (Scheer, Noweski y Meinel, 2012). De acuerdo con el plan de acción, se procedió a analizar resultados y discutir las conclusiones de los distintos actores de la comunidad, explorando el sentido que le habían dado a esta experiencia de aprendizaje:

Ha servido para que toda la clase podamos trabajar en una misma cosa... y así todo
el mundo tiene las cosas que quiere... nos hemos puesto de acuerdo para mejorar lo
de clase y hemos puesto cosas nuevas que nos pueden servir para que no sean las
mesas de siempre, para poder modernizar un poco. (Extracto 2: Intervención de
Blanca en la asamblea de valoración del proyecto)

Hemos aprendido a manejarnos con las piezas y saber cómo se enganchan y cómo se hacen... también las hemos hecho ajustables porque hay gente más alta y más baja así todos tienen una mesa a su medida. (Extracto 3: Intervención de Jara en la asamblea de valoración del proyecto)

Otra característica fundamental de la IAP es su condición de proceso recursivo, lo que deja abierta la posibilidad de iniciar un nuevo ciclo reflexivo y transformador, si la comunidad identifica nuevas necesidades en la fase de valoración:

En ocasiones, cuando los niños me ven en el colegio preguntan si vamos a hacer otro proyecto para fabricar más piezas y montar mesas para todos. En una de esas conversaciones coincidimos con el profesor y planteamos la posibilidad de diseñar otra dinámica para aprender cómo funciona la economía participativa, comúnmente asociada a la cultura digital, e intentar conseguir los recursos necesarios. (Extracto 
4: Notas de una conversación con Javier y Marcos, tutor y alumno de cuarto de primaria)

\section{Discusión y conclusiones}

Los desafíos para educar en la sociedad de la cultura digital han situado la investigación en un lugar crítico. Se trata de un momento de transformación profunda que exige a los profesionales de la enseñanza atender las necesidades de una sociedad que vive en un entorno de elevada incertidumbre. El rol del profesor en un futuro próximo probablemente le ubicará como profesional con un elevado grado de autogestión al que se requerirán competencias propias de un científico social (Kalantzis y Cope, 2012). El presente artículo se centra en situar y señalar ciertas cuestiones metodológicas de un estudio de caso que pretende ayudar a intervenir e innovar en procesos de cambio educativo, a la vez que se genera conocimiento, desde una perspectiva participativa y transdisciplinar propia de nuestra sociedad conectada. Se podría objetar a esta investigación la dificultad que plantea generalizar sus resultados frente a otros estudios que exploran contextos más claramente identificables y reconocibles (sean estos institucionalizados, formales o no). Sin embargo puede que ahí, precisamente, resida su interés, en su carácter complementario. Una búsqueda que se mueve en el intersticio de esos lugares con estructuras participativas complejas y dinámicas que se consensuan entre diferentes agentes socializadores, que aporta buena parte del corpus de datos evitando someterse a restricciones (aprovechando actividades con escasa concreción como las relacionadas con lo tecnológico en el caso del sistema escolar), sin violentarlas ni provocar rupturas (partiendo de prácticas o respetando ideologías socioeducativas que se examinan previamente en la fase de aproximación al campo).

Además de las relaciones interactivas de espacios (González-Patiño, 2011), siempre que las personas dispongan de la suficiente autonomía para desplegar su identidad social y cultural porque la disposición y decisiones de los agentes implicados así lo permitan (ideologías parentales, institución escolar, comunidad, convenciones, etc.), este abordaje sistémico va a hacer posible analizar el papel que juegan dichos agentes socializadores y las relaciones que se establecen entre ellos. Precisamente una de ellas, en apariencia la más controvertida desde el punto de vista metodológico, es el ya mencionado solapamiento de roles del investigador principal (padre de alumna, asesor y científico). Hay disponible literatura acerca de las contribuciones y limitaciones de la intervención familiar en procesos participativos de investigación en educación (Hurtig y Dyrness, 2011) y, como en el caso de esta IAP, plantea la conveniencia de adoptar la estrategia de incorporar investigadores no participantes para equilibrar el análisis.

Asimismo, se impone una reflexión crítica sobre la implicación de la familia en la escuela de la sociedad de la cultura participativa, teniendo presente además que los digitales son saberes discutidos sobre los que carecemos siquiera de un discurso experto de amplio consenso. Las familias producen desigualdades socioeducativas para favorecer a sus hijos dándoles oportunidades de las que no gozarán otras más vulnerables o en riesgo de exclusión, o incluso, las que no encajen en las categorías que definen los grupos dominantes (Bourdieu y Passeron, 2009; González-Patiño y Poveda, 2015). Precisamente, el discurso dominante en los medios de comunicación presenta como práctica parental responsable controlar, regular y limitar el uso de internet y redes sociales en la infancia (Hoover, Clark y Alters, 2004; Livingstone, Haddon y Görzig, 
2012), pero contrasta con la imagen emergente de una madre o un padre geek, fanático de la tecnología o de clase creativa, que juega a videojuegos con sus hijos, les acompaña y promueve un estilo de participación responsable en el medio digital, y que estaría detrás de una nueva élite del aprendizaje digital caracterizada por tipos específicos de capital social y cultural (Jenkins, Itó y Boyd, 2015). Esta podría ser la verdadera amenaza de la brecha digital, y no la conexión o el acceso a la tecnología, al menos durante los primeros años de vida de los niños que van a ser más dependientes de las ideologías socioeducativas de sus familias (Aliagas y Margallo, 2015). En este sentido el proyecto Arcángel :D, la comunidad educativa que participa en esta IAP, se situaría en línea con las iniciativas del programa de fondos digitales de identidad, una propuesta metodológica para que profesores, alumnos y sus familias, indaguen acerca de fondos culturales (artefactos o instrumentos culturales) y usos relevantes e intereses mediados por lo digital, con la intención de incorporarlos críticamente a sus prácticas educativas contribuyendo a convertirlas en un proceso transformador, significativo y sensible a las formas de vida de los niños (González-Patiño y Esteban-Guitart, 2015). La aspiración es propiciar un aprendizaje conectado (dentro y fuera de la escuela) que parte de la diversidad como fuente de eficiencia y garantía de sostenibilidad, y en el que el aprendiz es considerado co-diseñador (participación periférica legítima) de un aprendizaje entendido como una actividad situada y central en nuestra actual sociedad del conocimiento (Kalantzis y Cope, 2012).

Pero más allá de las discusiones teóricas, la modesta aportación de esta publicación consistiría en introducir un enfoque metodológico para la investigación educativa eminentemente aplicado, mostrando el trabajo que unos profesores dedicados, sus alumnos y las familias de estos, investigadores y profesionales, llevan a cabo transformando el aprendizaje, la forma de trabajar, la participación, la cultura y hasta la ciencia en su comunidad, tratando de interpretar hasta qué punto convergen estos cambios con los de otras experiencias concretas y analizando su correlato a escala global, con la aspiración de que compartir este conocimiento pueda inspirar el nuevo aprendizaje para la cultura digital.

\section{Agradecimientos}

La dedicación del primer autor e investigador principal ha sido parcialmente financiada por el programa de contratos predoctorales de la Universidad Autónoma de Madrid, convocatoria de 2011 . El proyecto de diseño y fabricación digital no hubiera sido posible sin la coordinación de Sara San Gregorio y su dedicación como mediadora en MedialabPrado Madrid, institución municipal que aportó recursos humanos y materiales de su fablab. Julio Albarrán y los miembros de su proyecto de investigación Cronicalab participaron documentando visualmente una buena parte de las sesiones, y Berta Gutiérrez, María Alonso, Marta San Gregorio, Manuel Jesús Bermúdez y Daniela Ricciardi, mediaron en la realización de talleres y actividades del proyecto. El claustro del colegio Arcángel y en particular Rosali García y Javier Díez, tutores de los grupos implicados, confiaron y fueron realmente flexibles con la toma de decisiones de esta compleja estructura de trabajo. Y las niñas y niños, auténticos agentes del cambio, siguen creciendo como investigadores empoderados y gracias a su aportación de conocimiento dotaron de sentido este estudio. 


\section{Referencias}

Aliagas, C. y Margallo, A. M. (2015). iPads, emergent readers and families. En M. Manresa y N. Real (Eds.), Digital literature for children: Text, readers and educational practices (pp. 155-172). Bruselas: Peter Lang.

Balcazar, F. E. (2003). Investigación acción participativa (IAP): Aspectos conceptuales y dificultades de implementación. Fundamentos en Humanidades, 4(8), 59-77.

Blikstein, P. (2013). Digital fabrication and making in education: The democratization of invention. En J. Walter-Herrmann y C. Büching (Eds.), FabLabs: Of machines, makers and inventors (pp. 1-21). Bielefeld: transcript Verlag.

Bourdieu, P. y Passeron, J.-C. (2009). Los herederos: los estudiantes y la cultura. Buenos Aires: Siglo Veintiuno Argentina.

Boyd, D. (2014). It's complicated: The social lives of networked teens. New Haven, CT: Yale University Press.

Castellá Sarriera, J. (2008). Análisis de las necesidades de un grupo o comunidad: la evaluación como proceso. En E. Saforcada y J. Castellá Sarriera (Eds.), Enfoques conceptuales y técnicos en psicología comunitaria (pp. 137-150). Argentina: Paidós.

Castells, M. (2009). Comunicación y poder. Madrid: Alianza.

Cobo, C. y Moravec, J. W. (2011). Aprendizaje invisible. Hacia una nueva ecología de la educación. Barcelona: Laboratori de Mitjans Interactius.

Erickson, F. (2014). Scaling down: A modest proposal for practice-based policy research in teaching. Education Policy Analysis Archives, 22(9), 1-11.

Fals Borda, O., Bonilla, V. D., Castillo, G. y Libreros, A. (1972). Causa popular, ciencia popular. Bogotá: La Rosca.

Freire, P. (1970). Pedagogía del oprimido. Ciudad de México: Siglo XXI.

Fundación Telefónica. (2016). La Sociedad de la información en España 2015. Barcelona: Ariel.

Gee, J. P. (2013). The anti-education era: creating smarter students through digital learning. Nueva York, NY: Palgrave Macmillan.

Goldin, C. D. y Katz, L. F. (2010). The race between education and technology. Boston, MA: Belknap Press of Harvard University Press.

González-Patiño, J. (2011). Rutinas de la infancia urbana mediadas por la tecnología: Un análisis visual. Papeles de Trabajo sobre Cultura, Educación y Desarrollo Humano, 7(2), 1-16.

González-Patiño, J. (2015). Familia, escuela y sociedad de la cultura digital: Construyendo comunidades educativas expandidas (Tesis doctoral) Universidad Autónoma de Madrid, Madrid.

González-Patiño, J. y Esteban-Guitart, M. (2014). Some of the challenges and experiences of formal education in a Mobile-Centric Society (MCS). Digital Education Review, 25, 64-86.

González-Patiño, J. y Esteban-Guitart, M. (2015). Fondos digitales de conocimiento e identidad: Un análisis etnográfico y visual. Papeles de Trabajo sobre Cultura, Educación y Desarrollo Humano, $11(2)$, 20-25.

González-Patiño, J. y Poveda, D. (2015). Privileging the individual through the collective commitment: Parental strategies and dynamics of involvement in a middle-class school. Multidisciplinary Journal of Educational Research, 5(3), 316-336. doi: $10.17583 /$ remie.2015.1524 
Hoover, S. M., Clark, L. S. y Alters, D. F. (2004). Media, home, and family. Nueva York, NY: Routledge.

Horst, H. A. y Miller, D. (2012). Digital anthropology. Londres: Berg.

Hurtig, J. y Dyrness, A. (2011). Parents as critical educators and ethnographers of schooling. En B. A. U. Levinson y M. Pollock (Eds.), A Companion to the anthropology of education (pp. 530-546). Oxford: Wiley-Blackwell.

Jenkins, H. (2008). Convergence culture: La cultura de la convergencia de los medios de comunicación. Barcelona: Paidós.

Jenkins, H., Ford, S. y Green, J. (2013). Spreadable media: Creating value and meaning in a networked culture. Nueva York, NY: New York University Press.

Jenkins, H., Itó, M. y Boyd, D. (2015). Participatory culture in a networked era: A conversation on youth, learning, commerce, and politics. Boston, MA: Polity Press.

Kalantzis, M. y Cope, B. (2012). New learning: Elements of a science of education. Nueva York, NY: Cambridge University Press.

Koh, J. H. L., Chai, C. S., Wong, B. y Hong, H. Y. (2015). Design thinking for education: conceptions and applications in teaching and learning. Nueva York, NY: Springer. doi:10.1007/978-981287-444-3

Lancy, D. F., Bock, J. C. y Gaskins, S. (2010). The anthropology of learning in childhood. Walnut Creek, CA: AltaMira Press.

Lave, J. y Wenger, E. (1991). Situated learning: legitimate peripheral participation. Nueva York, NY: Cambridge University Press.

Livingstone, S. M., Haddon, L. y Görzig, A. (2012). Children, risk and safety on the internet research and policy challenges in comparative perspective. Bristol: Policy Press.

Marsh, J. y Millard, E. (2006). Popular literacies, childhood and schooling. Nueva York, NY: Routledge.

Mediática. (2016a). Web de Mediática: Inspirando el nuevo aprendizaje para la cultura digital. Recuperado 10/12/2016, de http://mediatica.co/

Mediática. (2016b). Web del proyecto Arcángel :D, Acción digital escuela, AMPA y Mediática Recuperado 10/12/2016, de http://arcangel.mediatica.co/

Mitchell, C. (2011). Doing visual research. Londres: Sage Publications.

Pink, S. (2013). Doing visual ethnography: Images, media, and representation in research. Los Angeles, CA: SAGE.

Plowman, L., McPake, J. y Stephen, C. (2010). The technologisation of childhood? Young children and technology in the home. Children E Society, 24(1), 63-74.

Regina Frizzo, K. (2008). La investigación acción participante. En E. Saforcada y J. Castellá Sarriera (Eds.), Enfoques conceptuales y técnicos en psicología comunitaria (pp. 151-164). Buenos Aires: Paidós.

Scheer, A., Noweski, C. y Meinel, C. (2012). Transforming constructivist learning into action: Design thinking in education. Design and Technology Education: An International Journal, $17(3), 1-24$.

Thomson, P. (2010). Doing visual research with children and young people. Nueva York, NY: Routledge. 
Van Dijck, J. (2013). The culture of conectivity: A critical history of social media. Nueva York, NY: Oxford University Press.

Wardrop, A. y Withers, D. M. (2014). The para-academic handbook: a toolkit for making-learningcreating-acting. Bristol: HammerOn Press.

Wenger, E. (2005). Communities of practice: Learning, meaning, and identity. Cambridge: Cambridge University Press.

Wenger, E., McDermott, R. y Snyder, W. (2002). Cultivating communities of practice: A guide to managing knowledge. Boston, MA: Harvard Business School Press.

\section{Breve CV de los autores}

\section{Javier González-Patiño}

Doctor en Psicología por la Universidad Autónoma de Madrid y profesor en la Facultad de Formación de Profesorado y de Educación de la misma universidad. Con una dilatada experiencia como creativo digital, la intersección entre trayectoria profesional y formación académica orienta sus intereses de investigación, implicándose en proyectos participativos de tipo investigación acción en diferentes contextos socio-culturales, que permitan promover y analizar la mediación tecnológica en procesos educativos y de comunicación mediante una perspectiva interpretativa y transdisciplinar. Metodológicamente hablando, en su investigación predomina el enfoque cualitativo basado en estrategias de corte etnográfico con análisis narrativo y visual. ORCID ID: 0000-0003-3909-1897. Email: javier.gonzalezpatino@uam.es

\section{Moisés Esteban-Guitart}

Doctor en Psicología por la Universitat de Girona y profesor en la Facultad de Educación y Psicología de la misma universidad. Profesor invitado en el College of Education de la University of Arizona. Responsable del grupo de investigación "Cultura y Educación" y del Máster Interuniversitario en Psicología de la Educación - Doctorado Interuniversitario en Psicología de la Educación en la Universitat de Girona. Sus intereses de investigación se centran en el estudio de los mecanismos psicosociales implicados en la construcción de la identidad en contextos de diversidad social y cultural, y las relaciones familia, escuela y comunidad. Sus libros más recientes: "Funds of identity. Connecting meaningful learning experiences in and out of school" (2016, Cambridge University Press) y, con Ignasi Vila, "Familia, escuela y comunidad en las sociedades del siglo XXI" (2017, Horsori). ORCID ID: 0000-0002-1700-8792. Email: moises.esteban@udg.edu

\section{Sara San Gregorio}

Arquitecta por la Universidad Politécnica de Madrid. Responsable del grupo de investigación "A pequeña escala " en Medialab-Prado, que promueve la implicación infantil en los procesos de diseño y fabricación de sus objetos de juego, sus espacios de aprendizaje y sus experiencias ciudadanas. Centra su actividad en el diseño y producción de objetos mediadores del juego. y el aprendizaje dentro y fuera del aula colaborando con otros proyectos educativos basados en la experimentación como matemáticas visuales, 
objetos lectores, escala escuela. Es miembro del grupo Playgrounds con el que ha organizado dos encuentros sobre juegos y juguetes de construcción coordinando equipos multidisciplinares de expertos en diseño y en educación, en colaboración con la Universidad Autónoma de Madrid y Medialab-Prado. ORCID ID: 0000-0002-86333310 . Email: sarasangregoriodelucas@gmail.com 